Published in Numerical mathematics and advanced applications - ENUMATH 2013 Proceedings of ENUMATH 2013 : the 10th European Conference on Numerical Mathematics and Advanced Applications, Lausanne, August 2013. Cham : Springer, 2014 which should be cited to refer to this work

\title{
A Numerical Algorithm for a Fully Nonlinear PDE Involving the Jacobian Determinant
}

\author{
Alexandre Caboussat ${ }^{1}$ and Roland Glowinski ${ }^{2}$ \\ 1 Geneva School of Business Administration, University of Applied Sciences \\ Western Switzerland, Route de Drize 7, 1227 Carouge, Switzerland, \\ alexandre.caboussat@hesge.ch \\ 2 Department of mathematics, University of Houston, Houston, Texas, USA, \\ roland@math.uh.edu
}

\begin{abstract}
We address the numerical solution of the Dirichlet problem for a partial differential equation involving the Jacobian determinant in two dimensions of space. The problem consists in finding a vector-valued function such that the determinant of its gradient is given point-wise in a bounded domain, together with essential boundary conditions. The proposed numerical algorithm relies on an augmented Lagrangian algorithm with biharmonic regularization, and low order mixed finite element approximations. An iterative method allows to decouple the local nonlinearities and the global variational problem that involves a biharmonic operator. Numerical experiments validate the proposed method.
\end{abstract}

\section{Motivation}

Fully nonlinear equations can usually be written $F\left(\mathbf{u}, \nabla \mathbf{u}, \mathbf{D}^{2} \mathbf{u}\right)=0$, for some function $F$, in a bounded domain $\Omega$, together with Dirichlet boundary conditions. Several examples and numerical schemes can be found in $[1,2,5-7,9]$, applied mainly to second order equations, such as Monge-Ampère or Pucci's.

Inspired by $[3,4]$, we consider here a particular equation that involves only the Jacobian of the unknown function. Namely, for a given data $f$, we want to find $\mathbf{u}$ such that $\operatorname{det} \nabla \mathbf{u}=f$. This example has a geometric partial differential equation flavor, as it corresponds to finding a given deformation. Unlike for the Monge-Ampère equation, it thus does not involve the Hessian $\mathbf{D}^{2} \mathbf{u}$. The goal in the present work is to provide an alternative, for the computational viewpoint, to the theoretical, explicit, construction of solutions that exists in the literature for simple cases.

Following previous works on the Monge-Ampère equation [2], a variational approach is advocated. An iterative algorithm, reminiscent of alternating direction implicit methods, allows to alternatively solve linear variational problems and local nonlinear optimization problems. Numerical validation is achieved with simple examples, and convergence results are obtained from a computational perspective.

\section{Problem Formulation}

Let $\Omega$ be a bounded domain of $\mathbb{R}^{2}$, with $\Gamma$ its boundary, and $f: \mathbb{R}^{2} \rightarrow \mathbb{R}$ a given function. The fully nonlinear partial differential equation involving the Jacobian determinant we want to solve reads as follows: find $\mathbf{u}: \Omega \rightarrow \mathbb{R}^{2}$ satisfying 


$$
\begin{cases}\operatorname{det} \nabla \mathbf{u}=f & \text { in } \Omega \\ \mathbf{u}=\mathbf{I d} & \text { on } \Gamma\end{cases}
$$

where Id is the identity application. Problem (1) admits a solution, as discussed in $[3,4]$, under the compatibility condition on the data: $\int_{\Omega} f d \mathbf{x}=$ measure $(\Omega)$. Actually, the existence proof has been first made for $f \geq 0$ in [4], and extended to the more general case in [3]. However, the solution to (1) is not necessarily unique; indeed, for instance, if $\Omega$ is the unit disc and $f \equiv 1, \mathbf{u}_{1}(\mathbf{x})=\mathbf{x}$ is an obvious solution, and (denoting the polar coordinates by $(\rho, \theta)), \mathbf{u}_{2}(\rho, \theta)=$ $\left(\rho \cos \left(\theta+2 k \pi \rho^{2}\right), \rho \sin \left(\theta+2 k \pi \rho^{2}\right)\right)^{T}$ is also a solution.

We assume here that $f$ is non-negative. In order to design a numerical method based on some variational principle, and enforce the uniqueness of the solution to (1), we consider the following problem:

$$
\min _{\mathbf{v} \in \mathbf{E}} \frac{1}{2} \int_{\Omega}|\nabla \mathbf{v}-\mathbf{I}|^{2} d \mathbf{x}
$$

where $\mathbf{E}=\left\{\mathbf{v} \in H^{1}(\Omega)^{2}, \operatorname{det} \nabla \mathbf{v}=f,\left.\mathbf{v}\right|_{\Gamma}=\mathbf{I d}\right\}$. Here $\mathbf{I}$ denotes the $2 \times 2$ identity operator. The Fröbenius norm and product are respectively defined by $|\mathbf{T}|=(\mathbf{T}$ : $\mathbf{T})^{1 / 2}, \mathbf{S}: \mathbf{T}=\sum_{i, j=1}^{2} s_{i j} t_{i j}$ for each $\mathbf{S}=\left(s_{i j}\right), \mathbf{T}=\left(t_{i j}\right) \in \mathbb{R}^{2 \times 2}$. If $f \in L^{1}(\Omega)$, then the set $\mathbf{E}$ is not empty.

Let us denote by $\mathbf{u} \in \mathbf{E}$ the solution to (2). The choice of the objective distance function is arbitrary and is made in order to facilitate the decomposition properties of the algorithm discussed below.

\section{An Augmented Lagrangian Algorithm}

\subsection{Regularization and augmented Lagrangian functional}

We first introduce a biharmonic regularization to the variational problem (2). Let $\delta>0$ be a small parameter. The biharmonic regularization reads:

$$
\min _{\mathbf{v} \in \tilde{\mathbf{E}}}\left[\frac{1}{2} \int_{\Omega}|\nabla \mathbf{v}-\mathbf{I}|^{2} d \mathbf{x}+\frac{\delta}{2} \int_{\Omega}\left|\nabla^{2} \mathbf{v}\right|^{2} d \mathbf{x}\right]
$$

where $\tilde{\mathbf{E}}=\left\{\mathbf{v} \in H^{2}(\Omega)^{2}, \operatorname{det} \nabla \mathbf{v}=f,\left.\mathbf{v}\right|_{\Gamma}=\mathbf{I d}\right\}$. Then we introduce a new variable $\mathbf{p} \in L^{2}(\Omega)^{2 \times 2}$, so that (3) is equivalent to

$$
\min _{(\mathbf{v}, \mathbf{q}) \in \hat{\mathbf{E}}}\left[\frac{1}{2} \int_{\Omega}|\nabla \mathbf{v}-\mathbf{I}|^{2} d \mathbf{x}+\frac{\delta}{2} \int_{\Omega}\left|\nabla^{2} \mathbf{v}\right|^{2} d \mathbf{x}\right]
$$

where $\hat{\mathbf{E}}=\left\{\mathbf{v} \in H^{2}(\Omega)^{2}, \operatorname{det} \mathbf{q}=f,\left.\mathbf{v}\right|_{\Gamma}=\mathbf{I d}, \nabla \mathbf{v}=\mathbf{q}\right\}$. With formulation (4), we advocate an augmented Lagrangian algorithm. Namely, for $r>0$ a given parameter, we define the augmented Lagrangian functional

$$
\begin{aligned}
\mathcal{L}(\mathbf{v}, \mathbf{q} ; \mu)= & \frac{1}{2} \int_{\Omega}|\nabla \mathbf{v}-\mathbf{I} \mathbf{d}|^{2} d \mathbf{x}+\frac{\delta}{2} \int_{\Omega}\left|\nabla^{2} \mathbf{v}\right|^{2} d \mathbf{x} \\
& +\frac{r}{2} \int_{\Omega}|\nabla \mathbf{v}-\mathbf{q}|^{2} d \mathbf{x}+\int_{\Omega} \boldsymbol{\mu}:(\nabla \mathbf{v}-\mathbf{q}) d \mathbf{x} .
\end{aligned}
$$


and search for a saddle-point of $\mathcal{L}(\mathbf{v}, \mathbf{q} ; \mu)$. Thus, after defining the function spaces $\mathbf{V}=\left\{\mathbf{v} \in H^{2}(\Omega)^{2},\left.\mathbf{v}\right|_{\Gamma}=\mathbf{I} \mathbf{d}\right\}, \mathbf{Q}=\left\{\mathbf{q} \in L^{2}(\Omega)^{2 \times 2}\right\}$, and $\mathbf{Q}_{f}=\{\mathbf{q} \in \mathbf{Q}, \operatorname{det} \mathbf{q}=f\}$ the saddle-point problem consists in looking for $\{\mathbf{u}, \mathbf{p}, \boldsymbol{\lambda}\} \in \mathbf{V} \times \mathbf{Q}_{f} \times \mathbf{Q}$ such that

$$
\mathcal{L}(\mathbf{u}, \mathbf{p} ; \boldsymbol{\mu}) \leq \mathcal{L}(\mathbf{u}, \mathbf{p} ; \boldsymbol{\lambda}) \leq \mathcal{L}(\mathbf{v}, \mathbf{q} ; \boldsymbol{\lambda})
$$

for all $\{\mathbf{v}, \mathbf{q}, \boldsymbol{\mu}\} \in \mathbf{V} \times \mathbf{Q}_{f} \times \mathbf{Q}$.

\subsection{Iterative algorithm}

In order to solve (5), we advocate an Uzawa/alternating direction iterative algorithm. Let $\mathbf{u}^{0} \in \mathbf{V}$ and $\boldsymbol{\lambda}^{0} \in \mathbf{Q}$ be given. Then, for $n \geq 1$, we do:

(A) Solve the constrained nonlinear problem $\min _{\mathbf{q} \in \mathbf{Q}_{f}} \mathcal{L}\left(\mathbf{u}^{n-1}, \mathbf{p} ; \boldsymbol{\lambda}^{n-1}\right)$ to obtain $\mathbf{p}^{n} \in \mathbf{Q}_{f}$. This is equivalent to the quadratic problem under constraints:

$$
\min _{\mathbf{q} \in \mathbf{Q}_{f}}\left[\frac{r}{2} \int_{\Omega}|\mathbf{q}|^{2} d \mathbf{x}-\int_{\Omega} \mathbf{X}^{n-1}: \mathbf{q} d \mathbf{x}\right],
$$

where $\mathbf{X}^{n}:=r \nabla \mathbf{u}^{n}+\boldsymbol{\lambda}^{n} \in \mathbf{Q}$. This problem having no derivatives, it can be solved point-wise a.e. $\mathbf{x} \in \Omega$ (in practice on each element of a finite element discretization). Namely, for a.e. $\mathbf{x} \in \Omega$, it corresponds to a constrained quadratic problem: find $\mathbf{p}^{n}(\mathbf{x}) \in \mathbb{R}^{2 \times 2}$ solution of

$$
\min _{\mathbf{q} \in \mathbf{Q}_{\mathbf{x}}}\left[\frac{r}{2}|\mathbf{q}|^{2}-\mathbf{X}^{n-1}: \mathbf{q}\right],
$$

where $\mathbf{Q}_{\mathbf{x}}=\left\{\mathbf{q} \in \mathbb{R}^{2 \times 2}, \operatorname{det} \mathbf{q}=q_{11} q_{22}-q_{12} q_{21}=f(\mathbf{x})\right\}$.

(B) Solve the linear variational problem $\min _{\mathbf{v} \in \mathbf{V}} \mathcal{L}\left(\mathbf{v}, \mathbf{p}^{n} ; \boldsymbol{\lambda}^{n-1}\right)$ to obtain $\mathbf{u}^{n} \in \mathbf{V}$. This is equivalent to

$$
\min _{\mathbf{v} \in \mathbf{V}}\left[\frac{\delta}{2} \int_{\Omega}\left|\nabla^{2} \mathbf{v}\right|^{2} d \mathbf{x}+\frac{1+r}{2} \int_{\Omega}|\nabla \mathbf{v}|^{2} d \mathbf{x}-\int_{\Omega} \nabla \mathbf{v}: \mathbf{Y}^{n} d \mathbf{x},\right]
$$

where $\mathbf{Y}^{n}:=\mathbf{I}+r \mathbf{p}^{n}-\boldsymbol{\lambda}^{n-1} \in \mathbf{Q}$. This (linear) problem involves derivatives but does not include any constraints (other than the Dirichlet boundary conditions included in V). The first order optimality conditions corresponding to (8) lead to a linear variational problem, of the biharmonic type: find $\mathbf{u} \in \mathbf{V}$ satisfying

$$
\delta \int_{\Omega} \nabla^{2} \mathbf{u} \cdot \nabla^{2} \mathbf{v} d \mathbf{x}+(1+r) \int_{\Omega} \nabla \mathbf{u}: \nabla \mathbf{v} d \mathbf{x}=\int_{\Omega} \mathbf{Y}^{n}: \nabla \mathbf{v} d \mathbf{x},
$$

for all $\mathbf{v} \in\left(H^{2}(\Omega) \cap H_{0}^{1}(\Omega)\right)^{2}$.

(C) Update the multipliers $\boldsymbol{\lambda}^{n}=\boldsymbol{\lambda}^{n-1}+r\left(\nabla \mathbf{u}^{n}-\mathbf{p}^{n}\right) \in \mathbf{Q}$.

\subsection{Numerical solution of the constrained nonlinear problem}

Problem (7) can be rewritten as the following constrained finite dimensional minimization problem:

$$
\min _{\mathbf{q} \in \mathbf{E}_{c}}\left[\frac{1}{2}|\mathbf{q}|^{2}-\mathbf{b} \cdot \mathbf{q}\right]
$$


with $\mathbf{E}_{c}=\left\{\mathbf{q} \in \mathbb{R}^{4}, q_{1} q_{2}-q_{3} q_{4}=c(>0)\right\}$. Actually, here, $c=f(\mathbf{x})$ and $\mathbf{b}=$ $\frac{1}{r}\left(\mathbf{X}_{11}^{n}, \mathbf{X}_{22}^{n}, \mathbf{X}_{12}^{n}, \mathbf{X}_{21}^{n}\right)$. Problem (9) is solved with a Lagrangian approach and a Newton algorithm, after a suitable change of variables to take advantage of the structure of the problem (also encountered in incompressible finite elasticity, see, e.g., [8]). Let us denote by $\mathbf{S}$ the $4 \times 4$ orthogonal matrix

$$
\mathbf{S}=\left(\begin{array}{cccc}
1 / \sqrt{2} & 1 / \sqrt{2} & 0 & 0 \\
1 / \sqrt{2} & -1 / \sqrt{2} & 0 & 0 \\
0 & 0 & 1 / \sqrt{2} & 1 / \sqrt{2} \\
0 & 0 & 1 / \sqrt{2} & -1 / \sqrt{2}
\end{array}\right)
$$

and introduce the new variables $\mathbf{z}=\mathbf{S q}$, together with $\boldsymbol{\beta}=\mathbf{S b}$. Problem (9) is equivalent to

$$
\min _{\mathbf{z} \in \mathbf{F}_{c}}\left[\frac{1}{2}|\mathbf{z}|^{2}-\boldsymbol{\beta} \cdot \mathbf{z}\right],
$$

with $\mathbf{F}_{c}=\left\{\mathbf{z} \in \mathbb{R}^{4}, z_{1}^{2}-z_{2}^{2}-z_{3}^{2}+z_{4}^{2}=2 c(>0)\right\}$. In order to solve (10), let us introduce the associated Lagrangian functional $\mathcal{L}(\mathbf{z}, \mu)=\frac{1}{2}|\mathbf{z}|^{2}-\boldsymbol{\beta} \cdot \mathbf{z}-\frac{\mu}{2}\left(z_{1}^{2}-\right.$ $z_{2}^{2}-z_{3}^{2}+z_{4}^{2}-2 c$ ). If $\mathbf{y}$ is a solution of $(10)$, and $\lambda$ is a related Lagrange multiplier, the first order optimality conditions read:

$$
y_{1}=\frac{\beta_{1}}{1-\lambda}, y_{2}=\frac{\beta_{2}}{1+\lambda}, y_{3}=\frac{\beta_{3}}{1+\lambda}, y_{4}=\frac{\beta_{4}}{1-\lambda}, \frac{\beta_{1}^{2}+\beta_{4}^{2}}{(1-\lambda)^{2}}-\frac{\beta_{2}^{2}+\beta_{3}^{2}}{(1+\lambda)^{2}}=2 c
$$

It can be shown (see, e.g., [10]) that the solution of this system of equations corresponds to the unique solution of

$$
\frac{\beta_{1}^{2}+\beta_{4}^{2}}{(1-\lambda)^{2}}-\frac{\beta_{2}^{2}+\beta_{3}^{2}}{(1+\lambda)^{2}}=2 c
$$

that belongs to $(-1,+1)$. We then solve $(11)$ with a Newton method with initial guess $\lambda^{0}=0$.

Remark 1. In numerical experiments, the Newton method almost always converges to a root in $(-1,+1)$. When it is not the case, we arbitrarily set $\lambda=0$ and $y_{i}=$ $\beta_{i}, i=1, \ldots, 4$. This procedure does not jeopardize the convergence of the outer iterative algorithm.

\subsection{Numerical solution of the linear variational problem}

The first order optimality conditions related to (8) are: find $\mathbf{u}^{n+1} \in \mathbf{V}$ satisfying

$$
\delta \int_{\Omega} \nabla^{2} \mathbf{u}^{n+1} \cdot \nabla^{2} \mathbf{v} d \mathbf{x}+(1+r) \int_{\Omega} \nabla \mathbf{u}^{n+1}: \nabla \mathbf{v} d \mathbf{x}=\int_{\Omega} \mathbf{Y}^{n}: \nabla \mathbf{v} d \mathbf{x}
$$

for all $\mathbf{v} \in \mathbf{V}_{0}$, where $\mathbf{V}_{0}=\left\{\mathbf{v} \in H^{2}(\Omega)^{2},\left.\mathbf{v}\right|_{\Gamma}=\mathbf{0}\right\}$. Problem (12) is a classical biharmonic problem, closely related to those encountered when solving the elliptic Monge-Ampère equation in [2]. We observe that this problem is equivalent (if $\Omega$ 
is convex or $\partial \Omega$ smooth enough) to the following second-order variational system: find $\mathbf{w}^{n+1} \in\left(H_{0}^{1}(\Omega)\right)^{2}$ satisfying

$$
\delta \int_{\Omega} \nabla \mathbf{w}^{n+1}: \nabla \mathbf{v} d \mathbf{x}+(1+r) \int_{\Omega} \mathbf{w}^{n+1} \cdot \mathbf{v} d \mathbf{x}=\int_{\Omega} \mathbf{Y}^{n}: \nabla \mathbf{v} d \mathbf{x}, \quad \forall \mathbf{v} \in\left(H_{0}^{1}(\Omega)\right)^{2}
$$

followed with: find $\mathbf{u}^{n+1} \in\left(H^{1}(\Omega)\right)^{2},\left.\mathbf{u}^{n+1}\right|_{\partial \Omega}=\mathbf{g}$, satisfying

$$
\int_{\Omega} \nabla \mathbf{u}^{n+1}: \nabla \mathbf{v} d \mathbf{x}=\int_{\Omega} \mathbf{w}^{n+1} \cdot \mathbf{v} d \mathbf{x}, \quad \forall \mathbf{v} \in\left(H_{0}^{1}(\Omega)\right)^{2} .
$$

The solution of both second-order elliptic problems can be obtained with many well-known finite element techniques when $\Omega \subset \mathbb{R}^{2}$.

\section{Finite Element Approximation}

Finite elements are a natural choice for the discretization of (3) due to the variational flavor of this problem. A piecewise linear and globally continuous approximation of the solution $\mathbf{u}$ and piecewise constant approximations of its gradient $\nabla \mathbf{u}$ and of the additional variable $\mathbf{p}$ over a finite element triangulation of $\Omega$ are used here. Let $h>0$ be a discretization step, and $\mathcal{T}_{h}$ a conforming triangulation of $\Omega$. We assume for the sake of the discussion that $\Omega$ and $\Gamma$ are exactly approximated by their finite element discretizations. From $\mathcal{T}_{h}$, we approximate $\mathbf{Q}$ and $\mathbf{Q}_{f}$ respectively by

$$
\begin{aligned}
\mathbf{Q}_{h} & =\left\{\mathbf{q}_{h} \in L^{2}(\Omega)^{2 \times 2},\left.\mathbf{q}_{h}\right|_{T} \in \mathbb{R}^{2 \times 2}, \forall T \in \mathcal{T}_{h}\right\} \\
\mathbf{Q}_{f h} & =\left\{\mathbf{q}_{h} \in \mathbf{Q}_{h},\left.\operatorname{det} \mathbf{q}_{h}\right|_{T}=\bar{f}_{T}, \forall T \in \mathcal{T}_{h}\right\},
\end{aligned}
$$

where $\bar{f}_{T}$ is the value of the piecewise constant approximation of $f$ on $\mathcal{T}_{h}$ defined as $\bar{f}_{T}=\frac{1}{3} \sum_{T \ni P_{j}} f\left(P_{j}\right), P_{j}$ being the vertices of the triangle $T$. On the other hand, the space $\mathbf{V}$ is approximated by

$$
\mathbf{V}_{h}=\left\{\mathbf{v} \in C^{0}(\bar{\Omega})^{2},\left.\mathbf{v}\right|_{T} \in\left(\mathbb{P}_{1}\right)^{2}, \forall T \in \mathcal{T}_{h}, \mathbf{v}(\mathbf{x})=\mathbf{x} \text { on } \Gamma\right\},
$$

with $\mathbb{P}_{1}$ the space of the two-variables polynomials of degree $\leq 1$, and $\mathbf{I d}_{h}$ a piecewise linear interpolant of the identity function on $\Gamma$. Similarly, we define $\mathbf{V}_{0 h}=\left\{\mathbf{v} \in C^{0}(\bar{\Omega})^{2},\left.\mathbf{v}\right|_{T} \in\left(\mathbb{P}_{1}\right)^{2}, \forall T \in \mathcal{T}_{h}, \mathbf{v}(\mathbf{x})=\mathbf{0}\right.$ on $\left.\Gamma\right\}$.

The iterative algorithm in Section 3.2 can be re-written at the discrete level. The nonlinear optimization problem (6) is then solved element-wise on each triangle $T$ of $\mathcal{T}_{h}$, with exactly the same method as the one presented in Section 3.3 (when replacing $c:=f(\mathbf{x})$ by $\left.c:=\bar{f}_{T}\right)$. The discrete version of the variational problem (12) is solved with a sequence of discrete Poisson problems that are the discrete equivalents of (13) (14), in a similar fashion than in [2].

Remark 2 (On the choice of low order finite element approximations). The solution to (12) is actually a variation of the steady Stokes problem. Indeed, let us denote by $\mathbf{w}=\mathbf{v}-\mathbf{I d}$ and suppose that $\mathbf{w}$ is small and $f$ is close to 1 . We have then 


$$
\nabla \mathbf{w}=\nabla \mathbf{v}-\mathbf{I} \quad \operatorname{det} \nabla \mathbf{v}=\operatorname{det}(\mathbf{I}+\nabla \mathbf{w})=1+\nabla \cdot \mathbf{w}+\varepsilon(\mathbf{v})
$$

where $\varepsilon(\mathbf{w})$ is a (small) residual. This implies that $\nabla \cdot \mathbf{w}=(f-1)-\varepsilon(\mathbf{w})$, meaning that the vector field $\mathbf{w}$ is nearly divergence free. The problem we have to solve is thus closely related to the steady Stokes problem. Our approach, where $\mathbf{Q}_{h}$ is the space of the $2 \times 2$ matrix valued functions constant on each triangle $T$ of the triangulation $\mathcal{T}_{h}$ (used to approximate $\left(H^{1}(\Omega)\right)^{2}$ ), is therefore close to the $\mathbb{P}_{1}-\mathbb{P}_{1}$ approximation of the Stokes problem, which explains the convergence orders obtained in the next section.

\section{Numerical Validation}

Let us consider a validation case, for which (one of) the solution is the identity mapping $\mathbf{u}(\mathbf{x})=\mathbf{x}$. Thus, for the unit disc $\Omega=\left\{\mathbf{x} \in \mathbb{R}^{2},\|\mathbf{x}\|_{2}<1\right\}$, we consider: find $\mathbf{u}: \Omega \rightarrow \mathbb{R}^{2}$ satisfying

$$
\begin{cases}\operatorname{det} \nabla \mathbf{u}=1 & \text { in } \Omega \\ \mathbf{u}=\mathbf{I d} & \text { on } \Gamma\end{cases}
$$

The set of numerical parameters is given by $r=10^{-6}$ and $\delta=10^{-6}$. The tolerance between successive iterates $\mathbf{u}^{k}$ and $\mathbf{u}^{k+1}$ for the stopping criterion is set to $\varepsilon=$ $10^{-8}$. The tolerance for the Newton method for local nonlinear problems is set to $10^{-5}$ on the residual. The mesh is an unstructured Delaunay discretization of $\Omega$. The advocated numerical algorithm converges in less than 20 iterations (actually between 17 and 19 iterations depending on the mesh size). Figure 1 visualizes the solution on one given mesh (with $h \simeq 0.0161$ ). The most natural solution $\mathbf{u}(\mathbf{x})=$ $\mathbf{x}$ is correctly approximated, and the radial invariance is appropriately tracked even though the mesh does not guarantee such a symmetry. The determinant of $\mathbf{p}_{h}$ is exactly equal to one on each element (up to machine precision), while the determinant of $\nabla \mathbf{u}_{h}$ is nearly everywhere equal to one, implying that the constraint $\nabla \mathbf{u}_{h}=\mathbf{p}_{h}$ is weakly satisfied.

Numerical results are similar when considering the unit square $\Omega=(0,1)^{2}$, with either a structured or an unstructured mesh. Figure 1 (bottom right) illustrates the convergence of the error between the numerical solution $\mathbf{u}_{h}$ and the exact solution $\mathbf{u}=\mathbf{I d}$, for the unit disc and the unit square (with both types of meshes). All configurations lead to the convergence with order approximately $\mathcal{O}(h)$ of the numerical approximation towards the exact solution.

Future work will include more complicated test cases, including problems without solutions, non-convex domains, and the generalization to less regular Dirichlet boundary conditions.

\section{Acknowledgments}

The authors thank Prof. B. Dacorogna (EPFL) for suggesting the investigation of this problem, and for helpful comments and discussions. 

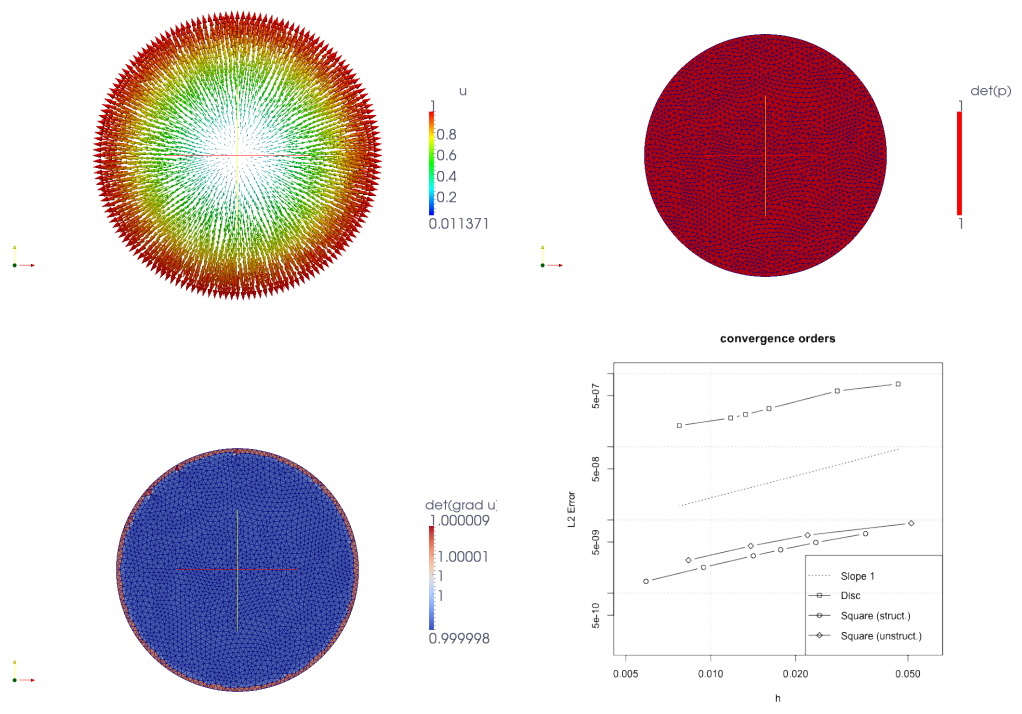

Fig. 1. Validation with the identity mapping. Visualization of the approximated solution obtained with the augmented Lagrangian approach after 19 steps $(h \simeq$ 0.0161). Vector field $\mathbf{u}_{h}$ (top left), determinant $\operatorname{det} \mathbf{p}_{h}$ (top right), determinant det $\nabla \mathbf{u}_{h}$ (bottom left), and (bottom right) convergence of the error in $L^{2}$ norm, for the unit disc and the unit square.

\section{References}

1. A. Caboussat, On the numerical solution of the Dirichlet problem for the elliptic $\left(\sigma_{2}\right)$ equation, Modeling, Simulation and Optimization in Science and Technology, vol. submitted, Springer, 2013.

2. A. Caboussat, R. Glowinski, And D. C. Sorensen, A least-squares method for the solution of the Dirichlet problem for the elliptic Monge-Ampère equation in dimension two, ESAIM: Control, Optimization and Calculus of Variations 19:3 (2013), 780-810.

3. G. Cupini, B. Dacorogna, And O. Kneuss, On the equation $\operatorname{det}(D u)=f$ with no sign hypothesis, Calculus of Variations and Partial differential Equations 36 (2009), 251-283.

4. B. Dacorogna And J. Moser, On a partial differential equation involving the Jacobian determinant, Ann. Inst. Henri Poincaré, Analyse Non Linéaire 7 (1990), 1-26.

5. X. Feng, R. Glowinski, And M. Neilan, Recent developments in numerical methods for fully nonlinear second order partial differential equations, SIAM Review 55:2 (2013), 205-267. 
6. X. Feng And M. NeIlan, Vanishing moment method and moment solutions for second order fully nonlinear partial differential equations, J. Sci. Comput. 38:1 (2009), 74-98.

7. B. D. Froese And A. M. Oberman, Convergent finite difference solvers for viscosity solutions of the elliptic Monge-Ampère equation in dimensions two and higher, SIAM J. Numer. Anal. 49:4 (2012), 1692-1714.

8. R. Glowinski AND P. L. TAlleC, Augmented Lagrangians and operatorsplitting methods in nonlinear mechanics, SIAM, Philadelphia, 1989.

9. O. LAKkis AND T. PRYER, A finite element method for fully nonlinear elliptic problems, SIAM J. Sci. Comput. 35:4 (2013), A2025-A2045.

10. D. C. Sorensen and R. Glowinski, A quadratically constrained minimization problem arising from PDE of Monge-Ampère type, Numer. Algor. 53:1 (2010), $53-66$. 